\title{
Marital quality, partner's engagement and continuous positive airway pressure adherence in obstructive sleep apnea
}

Short title: Marital relationship and CPAP adherence

Thibaut Gentina $\mathrm{MD}^{1 *}$, Sébastien Bailly $\mathrm{DPharm}, \mathrm{PhD}^{2,3 *}$, François Jounieaux $\mathrm{MD}^{1}$, Christophe Verkindre $M D^{4}$, Pierre-Marie Broussier $M D^{5}$, Dominique Guffroy $\mathrm{MD}^{6}$, Arnaud Prigent $\mathrm{MD}^{7}$, Jean-Jacques Gres $\mathrm{MD}^{8}$, Jamal Kabbani $\mathrm{MD}^{9}$, Laurent Kedziora $\mathrm{MD}^{10}$, Renaud Tamisier MD, PhD ${ }^{2,3}$, Elodie Gentina $\mathrm{PhD}^{11 \$}$, Jean-Louis Pépin $\mathrm{MD}^{2,3 \$}$

*These two first authors equally contributed to the work

\$These two senior authors equally contribute to the work

\section{Affiliations}

${ }^{1}$ Ramsey General Healthcare La Louviere Hospital, Lille, France

${ }^{2}$ HP2 Laboratory, INSERM U1042, Univ. Grenoble Alpes, Grenoble, France

${ }^{3}$ EFCR laboratory, Grenoble Alpes University Hospital, Grenoble, France

${ }^{4}$ Pneumology Department, Béthune Hospital, Béthune, France

${ }^{5}$ Pneumology Department, Clinique du Cèdre, Bois-Guillaume, France

${ }^{6}$ Pneumology practice, Maubeuge, France

${ }^{7}$ Saint Laurent Polyclinic, Rennes, France

${ }^{8}$ Pneumology Practice, Le Havre, France

${ }^{9}$ Pneumology Practice, Chelles, France

${ }^{10}$ Pneumology Department, Denain Hospital, Denain, France

${ }^{11}$ IESEG School of Management, LEM-CNRS (UMR 9221), Lille, France

\section{Corresponding author: Dr Thibaut Gentina}


Ramsay Générale de Santé Hôpital Privé La Louvière, 69 rue de la Louvière, 59000 Lille, France. email: t.gentina@wanadoo.fr.

\section{Authors' email adresses:}

t.gentina@wanadoo.fr; sbailly@chu-grenoble.fr; fjounieaux@gmail.com;

verkindre.christophe@gmail.com; pierre-marie.broussier@gmail.com;

dominique.guffroy@gmail.com; prigent@icloud.com; jean-jacques.gres@wanadoo.fr;

jamal.kabbani@wanadoo.fr; laukedz@gmail.com; rtamisier@chu-grenoble.fr;

e.gentina@ieseg.fr; jpepin@chu-grenoble.fr

Word count: main text 2424, abstract 260

Summary conflict of interest statements: Dr. Pépin reports grants from Philips, RESMED, Fisher and Paykel, Fondation de la recherche medicale, Direction de la recherche Clinique du CHU de Grenoble, Fond de dotation "Agir pour les maladies chroniques", and personal fees from Perimetre, Philips, Fisher and Paykel, RESMED, Astra-Zeneka and SEFAM, Agiradom, ELIA, Teva, during the conduct of the study. None of the other authors (TG, SB, FJ, CV, PMB, DG, AP, JJG, JK, LK, RT, EG) have anything to disclose.

Funding: This study was funded by unrestricted grant from ELIA Medical. SB, RT and JLP are supported by a research grant from the French National Research Agency (ANR-12-TECS0010), and in the framework of the "Investissements d'avenir" program (ANR-15-IDEX-02). 


\section{ABSTRACT}

Background: Continuous positive airway pressure (CPAP) is the first line therapy for obstructive sleep apnea (OSA) but its effectiveness requires high adherence. We aimed to assess the impacts of the spouse's/partner's involvement and the quality of the couple's relationship on CPAP adherence.

Methods: In a multicenter prospective study conducted in France, patients reported their subjective views regarding their partner's engagement in their CPAP treatment and the quality of their marital relationship using the Quality of Marriage Index. A hierarchical linear model was built to assess the predictors of CPAP adherence at day 120 . Structural equation modeling was performed to evaluate the direct and indirect effects of the spouse's/partner's engagement and the quality of the couple's relationship on CPAP adherence.

Results: The 290 OSA patients were predominantly male (77\%), with a median age of 53 years IQR: [46; 62], median BMI: $32 \mathrm{~kg} / \mathrm{m}^{2}$ [28.6; 35.9] and median apnea + hypopnea index: 43/per hour [33; 58]. Independent factors for CPAP adherence at day 120 were the partner's encouragement of CPAP usage and a stable relationship exceeding 30 years although emotional support or collaboration were not associated with CPAP adherence. Structural equation modeling demonstrated that spouse's/partner's engagement is directly related to CPAP adherence and improvement of symptoms, and that CPAP adherence is a mediator of disease-specific health-related quality of life. Marital quality was a significant moderator of these interactions meaning that a spouse's/partner's engagement improved adherence only when the quality of marriage index was high.

Conclusion: Future research and integrated OSA management should systematically include and document the role of the spouse/partner in CPAP adherence. 


\section{Keywords:}

CPAP adherence; partner engagement; marital quality; structural equation modeling; Obstructive sleep apnea treatment.

Abbreviations: AHI: apnea hypopnea index; BMI: Body Mass Index; CPAP: Continuous positive airway pressure; HST: home sleep test; IQR: Interquartile range; OSA: Obstructive sleep apnea; ODI: Oxygen desaturation Index; PSG: polysomnography; QMI: Quality of Marriage Index; QSQ: Quebec Sleep Questionnaire; SEM: Structural Equation Modelling. 


\section{INTRODUCTION}

Obstructive sleep apnea (OSA) is characterized by repetitive episodes of partial or complete pharyngeal obstruction during sleep [1]. OSA is one of the most frequent chronic diseases with both social and multi-organ consequences making it an economic burden for society $[1,2]$. OSA durably impairs the quality of life of patients and their entourage and is associated with co-morbidities including hypertension, arrhythmias, stroke, coronary heart disease and metabolic dysfunction [1]. Continuous positive airway pressure (CPAP), the first line therapy for OSA, needs to be used $>4 \mathrm{~h}$ per night to be effective in terms of symptom improvement and reduction of the burden of co-morbidities [3]. Yet, adherence to CPAP remains an issue for a significant percentage of OSA patients.

Interventions to improve CPAP adherence have included educational, supportive and behavioural strategies [4] or technical CPAP innovations to reduce device-related side effects. When implemented separately, these approaches only had a limited impact on CPAP adherence [4] and recent strategies have aimed at combining information and communications technologies (ICTs) for remote home monitoring of CPAP devices and patient coaching $[5,6]$. However, these approaches largely underestimate the pivotal role of the patient's domestic situation including the spouse's/partner's involvement and the quality of the, most frequently marital, partnership $[7,13]$.

To properly assess the role of couples in achieving good CPAP adherence, studies must now understand the couple as a psychosocial variable including compromises, communication, and social projects [14]. The quality of the couple's relationship, associated satisfaction $^{15}$ and the partner's involvement $[8,11,16,17]$ are the main items to be investigated when considering the couple's role in CPAP adherence. Previous studies in this field were frequently underpowered $[9,16]$ and included mainly or exclusively men with OSA 
$[8,10,11,16]$. There is a need to implement specific questionnaires assessing the different dimensions of how couples function and sophisticated statistical models to fully describe the integrative framework that motivates the relationship between partner involvement, the couple's satisfaction with their relationship and CPAP adherence. Finally, the relationship between improved CPAP usage, partner involvement and their effect on symptoms and quality of life requires further assessment.

Therefore, we performed a multicenter prospective observational study to assess the impact of marital quality, partner's engagement and a couple's characteristics on CPAP adherence at three months after CPAP initiation by unselected newly diagnosed OSA patients. We used structural equation modeling analysis to assess direct and indirect effects of partner involvement and the quality of the couple's relationship on CPAP adherence and patient centered outcomes.

\section{METHODS}

\section{Study participants}

Participants were recruited consecutively from May 2015 to December 2016, from eight private sleep centers in France. We used a definition of a couple that relies on the following three criteria [18]: (1) being over 18 years of age; (2) sharing the same principal residence for at least one year; and (3) declaring to be living as a couple or being married, or living under a civil or common-law union. Included subjects were patients newly diagnosed with OSA without any previous experience of CPAP usage. Standardized procedures for CPAP initiation and follow-up were established by the same homecare provider [Elia Médical, France] thus guaranteeing homogeneous practices for follow-up. We excluded patients with neurocognitive disorders or language fluency problems making them unable to complete 
study questionnaires. All patients provided written informed consent before participation, and the protocol was approved by the regional ethics Committee [Nord Ouest IV 013 A01842 43].

\section{OSA diagnosis and patient characterization}

OSA diagnosis was based on a full in-laboratory polysomnography (PSG) or a home sleep test (HST). Apneas, hypopneas, apnea hypopnea index (AHI) and micro-arousals were scored according to international recommendations $[19,20]$.

Relevant socio-demographic variables, Epworth Sleepiness Scale (ESS) and the Quebec Sleep Questionnaire (QSQ] [21] measuring patient reported disease-specific health-related quality of life, were collected.

\section{CPAP prescription, initiation and follow-up}

CPAP was prescribed to OSA patients with $\mathrm{AHI} \geq 30$ events per hour, or an $\mathrm{AHI}$ between 15 and 30 events per hour with symptoms and/or co-morbidities.

Before starting CPAP, patients benefited from a standardized one hour educational program including a 10-min video about OSA definitions, symptoms and the benefits of CPAP usage [22]. Objective CPAP adherence data were downloaded at day 120 from the CPAP device's software.

\section{Couples and quality of relationship characterization: Measure of partner's involvement}

At CPAP initiation, without the presence of their wife/husband/partner, patients evaluated their subjective view of their marital relationship using the Quality of Marriage Index (QMI) [23]. This was completed by additional items about the relationship including duration, family context and sleeping arrangements.

At day 45 after CPAP initiation, participants completed a questionnaire evaluating their partner's engagement regarding the CPAP treatment [11]. This covered three main domains 
of partner involvement: (i) pressure to use CPAP, (ii) emotional support, and (iii) collaboration in solving issues linked with CPAP usage. Details of the data collected are provided in the supplemental material (Appendix A).

\section{Statistical analysis}

Quantitative data are expressed as mean and standard deviation and qualitative variables are expressed as numbers and percentages.

\section{Factors associated with CPAP adherence at day 120}

We first performed a longitudinal data analysis using a hierarchical linear model, accounting for a potential center effect, to identify factors associated with CPAP adherence at day 120 . The hierarchical model was applied after verification of the assumption that CPAP adherence was normally distributed. An adjustment for confounding factors, selected after univariate analysis, was performed.

\section{Validation of interactions by structural equation modeling (SEM)}

Due to possible complex interactions and collinearity between measured variables generalized linear models were considered inadequate. To reinforce the reliability of our analysis, structural equation modeling (SEM) was used [24]. This method allows one to consider, in a unique model, collinear measurements by introducing latent variables. The SEM method is described in the Supplemental material (Appendix B).

\section{Hypotheses tested:}

Structural equation modeling allowed us to test the following five hypotheses (Figure 1): 


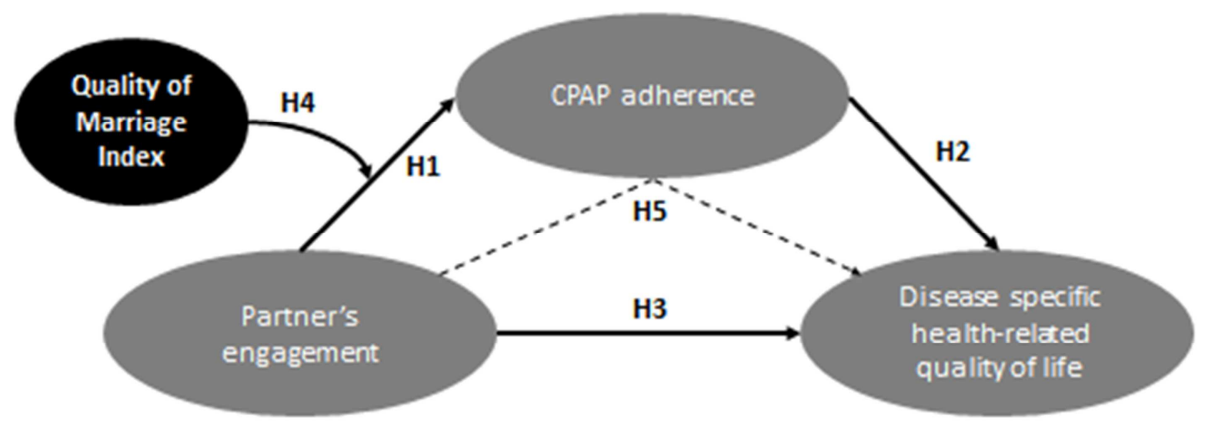

Hypothesis $1(\mathrm{H} 1)$ : Partner's engagement favoring CPAP use is positively related to the patient's CPAP adherence.

Hypothesis $2(\mathrm{H} 2)$ : CPAP adherence is a mediator of disease-specific health-related quality of life in CPAP-treated OSA patients.

Hypothesis $3(\mathrm{H} 3)$ : Partner's engagement is linked to disease-specific health-related quality of life in CPAP-treated OSA patients.

Hypothesis $4(\mathrm{H} 4)$ : The Quality of Marriage index is a moderator for the relationship between partner's engagement and CPAP adherence.

Hypothesis 5 (H5): CPAP adherence mediates the relationship between the partner's engagement and disease-specific health-related quality of life in CPAP-treated OSA patients. 
A group comparison was performed between two subgroups: low versus high quality of marriage index. Patients with a score $<3.20(\mathrm{~N}=131)$ were classed in the Low QMI group and patients with a QMI score $\geq 3.20(\mathrm{~N}=159)$ were classed in the high QMI group.

Statistical analyses were performed using SAS v9.4 (SAS NY Inc. Carry) and AMOS 21.0 (for SEM). Statistical significance was accepted when $p<0.05$ using two-tailed tests.

\section{RESULTS}

\section{Participant characteristics}

In total, 290 patients were included, mainly males (224, 77\%), with a median age of 53 years IQR: [46; 62], a median BMI of $32 \mathrm{~kg} / \mathrm{m}^{2}$ [28.6; 35.9] and a median Epworth Sleepiness score (ESS) of 11 [6; 15]. The baseline AHI was 43 events per hour [33; 58] and the baseline oxygen desaturation index (ODI) was 31.5 /h [18; 50] (Table 1).

\section{Table 1: Patient characteristics at baseline}

\begin{tabular}{ll}
\hline Variable & $\mathrm{N}=290$ \\
\hline Gender (Male) & $224(77.2)$ \\
Age, years & $53[46 ; 62]$ \\
Body mass index (BMI), kg/m² & $32.1[28.6 ; 35.9]$ \\
Professional status & \\
$\quad$ Active & $188(65.5)$ \\
Working at home & $6(2.1)$ \\
Unemployed & $14(4.9)$ \\
Retired & $79(27.5)$
\end{tabular}




\begin{tabular}{ll}
\hline Variable & $\mathrm{N}=290$ \\
\hline Apnea + hypopnea index (AHI) & $43[33 ; 58]$ \\
Oxygen desaturation index (ODI) & $31.5[18 ; 50]$ \\
Mean nocturnal $\mathrm{SpO}_{2}$ & $93[91 ; 94]$ \\
Epworth sleepiness score & $11[6 ; 15]$
\end{tabular}

Quantitative data are expressed in Median and interquartile range. Qualitative data are expressed in numbers and percentages.

\section{Couples}

The majority of couples were married $(246,85 \%)$ with a median duration of living together of 25 years [13; 35.5]. The partner frequently did not attend the initial diagnosis visit $(\mathrm{N}=75$, $26 \%$ ) but participated in the CPAP initiation session in $63 \%$ of cases. A minority of patients had night-time jobs ( $N=20,6.9 \%)$. The majority of couples had at least one child $(241,83 \%)$, and 188 couples had two or more children (65.3\%). Most couples slept in the same room $(\mathrm{N}=210,72.4 \%)$ and the size of the mattress was $140 \mathrm{~cm}$ in width for 112 couples (42\%) (Table 2).

Table 2: Couples' characteristics

\begin{tabular}{lr}
\hline Variable & $\mathrm{N}=290$ \\
\hline Duration of the couple (years) (median, IQR) & $25[13 ; 35.5]$ \\
Children (number (\%)) & $241(83.1)$ \\
Number of children &
\end{tabular}


Variable

$\mathrm{N}=290$

0

$47(16.3)$

1

$53(18.4)$

2

$106(36.8)$

$>2$

$82(28.5)$

Size of the mattress (number (\%))

$140 \mathrm{~cm}$

$112(41.8)$

$160 \mathrm{~cm}$

$>160 \mathrm{~cm}$

$68(25.4)$

Night-time work (patient) (number (\%))

$20(6.9)$

Night-time work (partner) (number (\%))

$11(3.8)$

Partner's engagement regarding CPAP treatment (median, IQR)

on scale of 5

Collaboration for solving issues linked with CPAP usage

$3.8[3.6 ; 5]$

Emotional support

$3.6[2.8 ; 4]$

Pressure to use CPAP

$3.8[3.4 ; 5]$

IQR : Interquartile range 


\section{Factors associated with CPAP adherence at day 120}

At day 120, median CPAP adherence was 359 minutes [307; 425] per night and the ESS score had improved to a median value of $6[3 ; 10]$. All five domains of the Quebec Sleep Questionnaire had significantly improved (Table 3). The percentage of woman was $22.7 \%$ $(\mathrm{N}=66)$. In this specific sub-group, poor CPAP adherence at 120 days was mostly associated with active professional activity. Retired or inactive women exhibited better CPAP compliance at 120 days.

Table 3: Quebec Sleep Questionnaire items measured at day 0 and day 120

\begin{tabular}{lccc}
\hline Variable & Day 0 & Day 120 & P value \\
\hline QSQ emotions & $2.6[2.0 ; 3.2]$ & $1.8[1.4 ; 2.4]$ & $<0.01$ \\
QSQ hypersomnolence & $2.7[1.8 ; 3.5]$ & $1.5[1.1 ; 2.2]$ & $<0.01$ \\
QSQ diurnal symptoms & $3.0[2.2 ; 3.6]$ & $1.7[1.2 ; 2.2]$ & $<0.01$ \\
QSQ nocturnal symptoms & $2.7[2.3 ; 3.4]$ & $1.9[1.4 ; 2.4]$ & $<0.01$ \\
QSQ social interaction & $2.0[1.3 ; 2.7]$ & $1.3[1.0 ; 1.7]$ & $<0.01$ \\
\hline
\end{tabular}

Univariate analysis using a mixed model and accounting for a potential center effect showed that the following parameters were significantly linked with CPAP adherence at day 120: partner encouraged CPAP usage, duration of couple's relationship and professional status (retired or not) (Supplementary Table 3 in supplementary material). After introducing the following variables: gender, $\mathrm{BMI}>30 \mathrm{~kg} / \mathrm{m}^{2}$, partner giving emotional support at day 45 , 
partner's encouragement of CPAP usage, duration of couple's relationship introduced as a qualitative variable (1-10 years, reference; $11-30$ years and $>30$ years), professional status, only two variables remained independent factors for CPAP compliance at day 120 in multivariate analysis: Encouragement of CPAP usage (estimate: $27.03+/-11.2, p=0.02$ ) and duration of the couple's relationship $>30$ years (estimate $=39.5+/-14.9, p=0.01$ ) compared to the shortest band (1-10 years).

\section{Structural Equation Modeling (SEM)}

\section{Figure 2: Structural Equation Modeling}

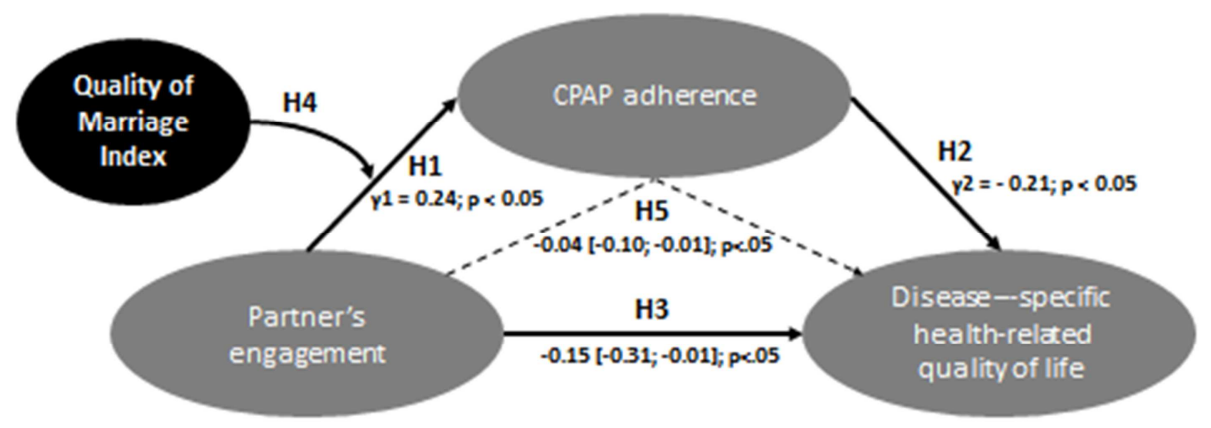

Hypothesis $1(\mathrm{H} 1)$ : Partner's engagement favoring CPAP use is positively related to the patient's CPAP adherence.

Hypothesis $2(\mathrm{H} 2)$ : CPAP adherence is a mediator of disease-specific health-related quality of life in CPAP-treated OSA patients. 
Hypothesis $3(\mathrm{H} 3)$ : Partner's engagement is linked to disease-specific health-related quality of life in CPAP-treated OSA patients.

Hypothesis $4(\mathrm{H} 4)$ : The Quality of Marriage index is a moderator for the relationship between partner's engagement and CPAP adherence.

Hypothesis 5 (H5): CPAP adherence mediates the relationship between the partner's engagement and disease-specific health-related quality of life in CPAP-treated OSA patients.

The final SEM revealed a positive and significant relationship between partner's engagement and the patient's CPAP adherence $(\gamma 1=0.24 ; \mathrm{p}<0.05)$, in support of $\mathrm{H} 1$ (Figure 2). The level of CPAP adherence was significantly linked with disease-specific health-related quality of life improvement $(\gamma 2=-0.21 ; p<0.05)$, validating $\mathrm{H} 2$. Also, partner's engagement directly impacted on improvement in disease-specific health-related quality of life in patients with OSA $(\gamma 3=-0.15 ; p<0.05)$, in support of H3. In testing the mediation effects (Table 4$)$, the quality of marriage index (QMI) revealed that the relationship between a partner's involvement and the patient's CPAP adherence was significant only for patients having a high QMI ( $\gamma 4 a=0.32 ; p<0.001)$ but not for those with low QMI $(\gamma 4 b=0.02 ; p=0.82)$. The $\chi^{2}$ difference values reached significance $(p<0.04)$, in support of $H 4$. Thus, the quality of marriage plays a key role in the relationship between spousal involvement and patients CPAP adherence. Finally, the mediation effects of the whole sample reached consistent results, validating H5 (Table 4; Figure 2). 


\begin{tabular}{|c|c|c|c|c|c|}
\hline & $\begin{array}{c}\text { Standardized } \\
\text { parameter } \\
\text { estimates }\end{array}$ & $\begin{array}{c}\text { Bootstrapped } \\
\text { confidence } \\
\text { interval }\end{array}$ & $\begin{array}{c}P \\
\text { Value }\end{array}$ & $\begin{array}{c}\text { Mediatio } \\
n\end{array}$ & Results \\
\hline \multicolumn{6}{|l|}{ Whole sample $(n=290)$} \\
\hline $\begin{array}{l}\text { Direct effect (H3): } \\
\text { Partner's engagement } \\
\rightarrow \text { Disease-specific } \\
\text { health-related quality } \\
\text { of life in patients }\end{array}$ & -0.15 & {$[-0.31 ;-0.01]$} & $<0.05$ & Partial & $\begin{array}{c}\text { H3 } \\
\text { Supported }\end{array}$ \\
\hline $\begin{array}{l}\text { Indirect effect }(\mathbf{H} 5) \text { : } \\
\text { Partner's engagement } \\
\rightarrow \text { CPAP use } \rightarrow \\
\text { Disease-specific health- } \\
\text { related quality of life in } \\
\text { patients }\end{array}$ & -0.04 & {$[-0.10 ;-0.01]$} & $<0.05$ & & $\begin{array}{c}\text { H5 } \\
\text { supported }\end{array}$ \\
\hline
\end{tabular}

\section{DISCUSSION}

We demonstrated that partner's involvement and the quality of the couple's relationship significantly impact on CPAP adherence and the perception of improvement with therapy (eg, Quality of life). Our data are supported by robust structural equation modeling demonstrating that partner's involvement improves CPAP adherence which indirectly participates towards improvement of health related quality of life and patient centered outcomes. However, this was true only in the subgroup of couples reporting a high quality of marriage index. 
Marital status can be simply characterized as a socio-demographic variable potentially impacting CPAP adherence. It has been reported that OSA patients living alone exhibited poor CPAP adherence $[7,25]$ compared to those who were married or living as a couple [26]. Sharing the same bed also has a positive effect on male OSA patients' CPAP adherence [27]. In most previous studies, the couple was considered as a homogeneous socio-demographic variable that was essentially studied as one of the determinants of CPAP adherence. The novelty of our study is to provide new knowledge by considering the different psychological and social dimensions of the relationship and precisely characterizing the impact of the relationship's quality on CPAP adherence. The majority of previous studies showed that perceived spousal involvement (collaboration and support) contributed to increase CPAP adherence among men with severe OSA $[11,16]$. In line with this, in the APPLES study the level of spousal involvement was significantly linked with increased CPAP adherence at six months but only in males [17]. However, they found no significant differences in overall marital quality when comparing adherent and non-adherent CPAP-treated OSA patients using an arbitrary threshold of 4 hours/night. While in our study, results were adjusted for gender, CPAP adherence was evaluated only at three months. The long term three-year follow-up in the APPLES study [17] suggested that the level of spousal involvement was significant at CPAP initiation but was not predictive of longer-term adherence. Probably, in couples with a poor relationship CPAP withdrawal frequently occurs in the first months after CPAP initiation meaning that a specific management strategy needs to be implemented in this context. Compared to previous studies with relatively small sample sizes $[9,16]$ or exclusively men $[8,10,11,16]$ our study certainly provides results with a higher potential for generalizability in the routine practice of CPAP initiation. 
Neither the partner's emotional support nor collaboration was significantly associated with CPAP adherence. Only pressure to use CPAP from the partner mattered and when the partner was really insistent, the patients used their CPAP more. This could easily be seen as nagging or passive-aggressive behavior that can become contra-productive in those with lower QMI.

Another strength of our work was to use structural equation modeling (SEM) allowing for the first time to define the respective roles of partner's involvement and the quality of the relationship (quality of marriage index, QMI). The SEM analysis showed a significant direct effect of the partner involvement in disease-specific health-related quality of life in CPAP-treated OSA patients. The impact of improved CPAP adherence on symptoms and quality of life remains unclear and heterogeneous between studies [4], and requires clarification. In the network meta-analyses of Bratton et al., $[28,29]$ looking at the effects of CPAP on sleepiness and quality of life, the meta-regression analysis did not provide evidence that studies reporting CPAP adherence also reported larger treatment effects. Different clusters of OSA phenotypes are associated with different levels of CPAP adherence. In our study, somnolence was considered as an outcome and not an explicative factor. Further studies are needed to assess the impact of the quality of a couple's relationship in specific OSA phenotypes and in particular in minimally symptomatic patients. Our results suggest that beyond the improvement generated by CPAP itself, the perception of benefits in terms of quality of life is largely mediated by the couple's context. Another structural equation model demonstrated that CPAP adherence improved the intimate relationship between bed partners directly and indirectly, and the effect was mediated through reduced daytime sleepiness and increased activity levels [10]. The QMI was introduced in the structural equation model as a moderator but this does not allow one to make a causal interpretation 
of the impact of the quality of the marriage on CPAP adherence. It should only be considered as an interesting variable to bear in mind in routine clinical practice. The same personal characteristics facilitating a good relationship might also lead to high CPAP adherence. Again, this underscores the importance of the triangle describing the quality of a couple's relationship, CPAP adherence and the evolution of patient centered outcomes.

According to Baron et al patients report diverse perceptions of spousal involvement in CPAP initiation and tolerance [16]. In our study, marital quality index was demonstrated to be a mediator between partner involvement and CPAP adherence. Positive partner involvement including supportive and collaborative interventions has the potential to improve CPAP adherence, whereas inappropriate pressure might not be beneficial.

\section{Conclusion and perspectives}

CPAP adherence should be supported by a broader understanding of the patient's affective environment by including a dyadic perspective encompassing patients, their partners and the quality of the relationship. ${ }^{8}$ Further research is needed to test supportive spousal involvement as an adherence intervention for CPAP treated OSA patients.

\section{ACKNOWLEDGEMENTS}

We thank Dr. Bernard Douay for data collection and study monitoring and Dr Alison Foote (Grenoble Alpes University Hospital) for critically editing the manuscript.

\section{Authors' contributions:}

Conception and design: TG, SB, EG, JLP, Inclusion of patients and collection of data: TB, FJ, CV, PMB, DG, AP, JJG, JK, LK, Analysis and interpretation: SB, RT, JLP, Drafting the 
manuscript: TG, SB, RT, EG, JLP, Critically revising the manuscript: FJ, CV, PMB, DG, AP, JJG, JK, LK,

JLP had full access to all of the data in the study and takes responsibility for the integrity of the data and the accuracy of the data analysis

All authors have read and approved the submitted manuscript.

\section{Role of the funding source:}

None of the funding sources were involved in study design, in the collection, analysis and interpretation of data, in the writing of the report, or in the decision to submit the article for publication. 


\section{REFERENCES}

1. Levy P, Kohler M, McNicholas WT et al. Obstructive sleep apnoea syndrome. Nat Rev Dis Primers 2015; 1: 15015.

2. Watson NF. Health Care Savings: The Economic Value of Diagnostic and Therapeutic Care for Obstructive Sleep Apnea. J Clin Sleep Med 2016; 12: 1075-7.

3. Javaheri S, Barbe F, Campos-Rodriguez F, et al. Sleep Apnea: Types, Mechanisms, and Clinical Cardiovascular Consequences. J Am Coll Cardiol 2017; 69: 841-58.

4. Wozniak DR, Lasserson TJ, Smith I. Educational, supportive and behavioural interventions to improve usage of continuous positive airway pressure machines in adults with obstructive sleep apnoea. Cochrane Database Syst Rev 2014: CD007736.

5. Pepin JL, Tamisier R, Hwang D, Mereddy S, Parthasarathy S. Does remote monitoring change OSA management and CPAP adherence? Respirology 2017; 22: 1508-17.

6. Hwang D, Chang JW, Benjafield AV, Crocker ME, Kelly C, Becker KA, Kim JB, Woodrum RR, Liang J, Derose SF. Effect of Telemedicine Education and Telemonitoring on Continuous Positive Airway Pressure Adherence. The Tele-OSA Randomized Trial. Am J Respir Crit Care Med 2018; 197: 117-26.

7. Poulet C, Veale D, Arnol N, et al. Psychological variables as predictors of adherence to treatment by continuous positive airway pressure. Sleep Med 2009; 10: 993-9.

8. Ye L, Malhotra A, Kayser K, et al. Spousal involvement and CPAP adherence: a dyadic perspective. Sleep Med Rev 2015; 19: 67-74.

9. Luyster FS, Dunbar-Jacob J, Aloia MS,et al. Patient and Partner Experiences With Obstructive Sleep Apnea and CPAP Treatment: A Qualitative Analysis. Behav Sleep Med 2016; 14: 67-84.

10. Lai AY, Ip MS, Lam JC, et al. A pathway underlying the impact of CPAP adherence on intimate relationship with bed partner in men with obstructive sleep apnea. Sleep Breath 2016; 20: $543-51$. 
11. Baron KG, Smith TW, Berg CA, et al. Spousal involvement in CPAP adherence among patients with obstructive sleep apnea. Sleep Breath 2011; 15: 525-34.

12. Crawford MR, Espie CA, Bartlett DJ, Grunstein RR. Integrating psychology and medicine in CPAP adherence--new concepts? Sleep Med Rev 2014; 18: 123-39.

13. McDowell A. Spousal involvement and CPAP adherence: a two-way street? Sleep Breath 2011; 15: 269-70.

14. Rosenblatt PC. Two in a Bed: The Social System of Couple Bed Sharing. Albany: State University of New York Press; 2006.

15. Baron KG, Smith TW, Czajkowski LA, et al. Relationship quality and CPAP adherence in patients with obstructive sleep apnea. Behav Sleep Med 2009; 7: 22-36.

16. Glazer-Baron K, Gunn HE, Czajkowski LA, et al. Spousal involvement in CPAP: does pressure help? J Clin Sleep Med 2012; 8: 147-53.

17. Batool-Anwar S, Baldwin CM, Fass S, et al. Role of Spousal Involvement in Continuous Positive Airway Pressure (Cpap) Adherence in Patients with Obstructive Sleep Apnea (Osa). Southwest J Pulm Crit Care 2017; 14: 213-27.

18. Merenda A, Miano P. Co-parental Couples and New Families: A Study of the Primary Triad. Procedia - Social and Behavioral Sciences 2015; 174: 1107-10.

19. Budhiraja R, Parthasarathy S, Drake CL, et al. Early CPAP use identifies subsequent adherence to CPAP therapy. Sleep 2007; 30: 320-4.

20. Berry RB, Budhiraja R, Gottlieb DJ, et al. Rules for scoring respiratory events in sleep: update of the 2007 AASM Manual for the Scoring of Sleep and Associated Events. Deliberations of the Sleep Apnea Definitions Task Force of the American Academy of Sleep Medicine. J Clin Sleep Med 2012; 8: 597-619.

21. Lacasse Y, Bureau MP, Series F. A new standardised and self-administered quality of life questionnaire specific to obstructive sleep apnoea. Thorax 2004; 59: 494-9. 
22. Jean Wiese H, Boethel C, Phillips B, et al. CPAP compliance: video education may help! Sleep Med 2005; 6: 171-4.

23. Norton R. Measuring Marital Quality: A Critical Look at the Dependent Variables. Journal of Marriage and Family 1983; 45: 141-51.

24. Kline RB. Principles and Practice of Structural Equation Modeling, $3^{\text {rd }}$ Edition (Methodology in the Social Sciences) Guilford Press; 2011.

25. Lewis KE, Seale L, Bartle IE, et al. Early predictors of CPAP use for the treatment of obstructive sleep apnea. Sleep 2004; 27: 134-8.

26. Gagnadoux F, Le Vaillant M, Goupil F, et al. Influence of marital status and employment status on long-term adherence with continuous positive airway pressure in sleep apnea patients. PLoS One 2011; 6: e22503.

27. Cartwright R. Sleeping together: a pilot study of the effects of shared sleeping on adherence to CPAP treatment in obstructive sleep apnea. J Clin Sleep Med 2008; 4: 123-7.

28. Bratton DJ, Gaisl T, Schlatzer C, et al. Comparison of the effects of continuous positive airway pressure and mandibular advancement devices on sleepiness in patients with obstructive sleep apnoea: a network meta-analysis. Lancet Respir Med 2015; 3: 869-78.

29. Kuhn E, Schwarz El, Bratton DJ, et al. Effects of CPAP and Mandibular Advancement Devices on Health-Related Quality of Life in OSA: A Systematic Review and Meta-analysis. Chest 2017; 151: 786-94. 